\title{
LETTERS: NEW OBSERVATIONS
}

\section{Web-Based Assessment of Parkinson's Prodromal Markers Identifies GBA Variants}

Identifying those "at risk" of Parkinson's disease (PD) is a priority and a key strategy for testing neuroprotective agents. ${ }^{1}$ PREDICT-PD is a pilot cohort study in which healthy UK adults are screened online (www.predictpd.com) with a questionnaire that ascertains the presence of early phenotypic features and risk factors, based on systematic review. ${ }^{2}$ Subjects are ascribed risk scores according to their answers and then complete additional online tests. ${ }^{3}$ Higherrisk and lower-risk subjects are compared by using various markers for PD and eventual clinical outcome. We hypothesized that if risk stratification were effective in 1,319 eligible participants, an excess of GBA variants and LRRK2 mutations would be found in higher-risk subjects compared with lower-risk (inclusion and exclusion criteria and risk scoring have been previously described ${ }^{3}$ ). A greater likelihood of identifying differences in outcomes was anticipated if extremes of risk were sampled. Risk scores were ranked to enable comparative analyses between subjects with the highest $15 \%$ and lowest $15 \%$ risk scores. Invitations were extended to these subjects, and additional subjects sampled randomly from the middle-risk group, to participate with inperson clinical studies (see Supplemental Data Table for sampling and acceptance rates).

Sanger sequencing was performed on DNA extracted from the saliva of 192 subjects to screen exon 41 of $L R R K 2$ (which contains the commonest c.6055 G>A; p.G2019S mutation) and exons 8-11 of the GBA gene (contains almost $90 \%$ of recognized GBA pathogenic variants). ${ }^{4} G B A$ variants were found in 6 of 75 higher- $(1$ N370S, 2 E326K, 3 T369M), 0 of 43 middle-, and 1 of 67 lower-risk subjects

*Correspondence to: Professor John Hardy, Department of Molecular Neuroscience and Reta Lila Weston Institute, UCL Institute of Neurology, 1 Wakefield Street, London WC1N 1PJ, UK, e-mail: j.hardy@ucl.ac.uk

Relevant conflicts of interest/financial disclosures: Nothing to report. Full financial disclosures and author roles may be found in the online version of this article.

Funding agencies: The PREDICT-PD pilot study and A.J.N. are funded by Parkinson's UK (grant references K-1006 and F-1201). This study was also supported by the Wellcome Trust/Medical Research Council Joint Call in Neurodegeneration award (WT089698) to the Parkinson's Disease Consortium (UKPDC), whose members are from the UCL Institute of Neurology, University of Sheffield, and the Medical Research Council Protein Phosphorylation Unit at the University of Dundee. The work was also supported by the National Institute for Health Research University College London Hospitals Biomedical Research Centre.

${ }^{\dagger}$ Both authors contributed equally to this work.

Received: 12 January 2015; Revised: 24 March 2015; Accepted: 29 March 2015

Published online 13 May 2015 in Wiley Online Library (wileyonlinelibrary.com). DOI: 10.1002/mds.26249
TABLE 1. Participants, proportion tested, and mutations in groups

\begin{tabular}{lcccc}
\hline & $\begin{array}{c}\text { Higher-Risk } \\
\text { Participants }\end{array}$ & $\begin{array}{c}\text { Middle-Risk } \\
\text { Participants }\end{array}$ & $\begin{array}{c}\text { Lower-Risk } \\
\text { Participants }\end{array}$ & $P$-Value \\
\hline $\begin{array}{c}\text { Total subjects } \\
\text { per group }\end{array}$ & 195 & 926 & 198 & \\
$\begin{array}{c}\text { Number that } \\
\text { underwent }\end{array}$ & 79 & 45 & 68 & \\
$\quad \begin{array}{l}\text { screening } \\
\begin{array}{c}\text { Sequencing } \\
\text { failures }\end{array}\end{array}$ & 4 & 2 & 1 & \\
$\begin{array}{c}\text { Sequenced } \\
\text { (\% of total group) }\end{array}$ & $75(38)$ & $43(5)$ & $67(34)$ & \\
$\begin{array}{l}\text { GBA variants } \\
\text { LRRK2 G2019S }\end{array}$ & 6 & 0 & 1 & $0.018^{\mathrm{a}}$ \\
mutations & 0 & 0 & 0 & - \\
\hline
\end{tabular}

${ }^{\text {a}}$ Fisher's exact test.

(T369M homozygous) (see Table 1). The odds ratio of having a $G B A$ variant in the higher- versus the pooled middleand lower-risk groups was 9.48 (95\% confidence interval, 1.12-80.47; $P=0.018)$. LRRK2 G2019S mutations were not found in any participant.

Of the variants identified, N370S has a deleterious effect on glucocerebrosidase enzyme activity and is known to cause Gaucher's disease, as well as being a clear risk factor for PD. E326K and T369M are functionally mild variants, not severe enough to cause Gaucher's disease even when present in the homozygous/compound heterozygous state. ${ }^{5,6}$ Nonetheless, our results add to the notion that both E326K and T369M may, in the right milieu of genetic and environmental factors, contribute to the risk of PD. ${ }^{4,5}$ Failure to find LRRK2 mutations was unsurprising given the rarity of the G2019S mutation in healthy controls and its presence in only $1 \%$ to $2 \%$ of patients with $\mathrm{PD}^{7}$ GBA variants were found in $8 \%$ of higher-risk subjects, a proportion similar to estimates of frequency in sporadic PD. ${ }^{4}$ Conversely, less than $1 \%$ of lower-risk subjects were found to have a GBA variant. An overall rate of $3.6 \%$ in the total sample was observed, which is similar to the $4 \%$ reported in healthy controls. ${ }^{5}$ Age is a powerful determinant of PD risk but has the potential to confound given its effect on many outcome measures used in predictive studies (eg, sense of smell, objective motor function, and functional imaging). By using GBA variants as an outcome for risk stratification, age-independent support for enrichment was observed.

Alastair J. Noyce, MRCP, $\dagger^{1,2}$ Niccolo E. Mencacci, MD,$\dagger^{2}$ Anette Schrag, FRCP, PhD, ${ }^{3}$ Jonathan P. Bestwick, MSc, ${ }^{4}$ 
Gavin Giovannoni, FRCP, PhD ${ }^{5}$ Andrew J. Lees, FRCP, $\mathrm{MD},{ }^{1,2}$ and John Hardy, PhD*1,2

${ }^{1}$ Reta Lila Weston Institute, UCL Institute of Neurology

${ }^{2}$ Department of Molecular Neuroscience, UCL Institute of Neurology

${ }^{3}$ Department of Clinical Neuroscience, UCL Institute of Neurology

${ }^{4}$ Wolfson Institute of Preventive Medicine, Queen Mary University of London

${ }^{5}$ Blizard Institute, Barts and the London School of Medicine and Dentistry, Queen Mary University of London

\section{References}

1. Sieber B-A, Landis S, Koroshetz W, et al. Prioritized research recommendations from the National Institute of Neurological Disorders and Stroke Parkinson's Disease 2014 conference. Ann Neurol 2014;76:469-472.

2. Noyce AJ, Bestwick JP, Silveira-Moriyama L, et al. Meta-analysis of early nonmotor features and risk factors for Parkinson disease. Ann Neurol 2012;72:893-901.

3. Noyce AJ, Bestwick JP, Silveira-Moriyama L, et al. PREDICT-PD: identifying risk of Parkinson's disease in the community: methods and baseline results. J Neurol Neurosurg Psychiatry 2014;85:31-37.

4. Winder-Rhodes SE, Evans JR, Ban M, et al. Glucocerebrosidase mutations influence the natural history of Parkinson's disease in a community-based incident cohort. Brain 2013;136:392-399.

5. Duran R, Mencacci NE, Angeli AV, et al. The glucocerobrosidase E326K variant predisposes to Parkinson's disease, but does not cause Gaucher's disease. Mov Disord 2012;28:232-236.

6. Walker JM, Lwin A, Tayebi N, et al. Glucocerebrosidase mutation T369M appears to be another polymorphism. Clin Genet 2003;63: 237-238.

7. Healy DG, Falchi M, O'Sullivan SS, et al. Phenotype, genotype, and worldwide genetic penetrance of LRRK2-associated Parkinson's disease: a case-control study. Lancet Neurol 2008;7:583-590.

\section{Supporting Data}

Additional Supporting Information may be found in the online version of this article.

\section{Adaptive Deep Brain Stimulation in a Freely Moving Parkinsonian Patient}

The future of deep brain stimulation (DBS) for Parkinson's disease (PD) lies in new closed-loop systems that continuously supply the implanted stimulator with new settings obtained by analyzing a feedback signal related to the patient's current clinical condition. ${ }^{1}$ The most suitable feedback for PD is subthalamic local field potential (LFP) activity recorded from the stimulating electrode itself. $^{2-4}$ This closed-loop technology known as adaptive DBS (aDBS) recently proved superior to conventional open-loop DBS (cDBS) in patients with PD. ${ }^{2}$

No studies have yet tested aDBS in freely moving humans for a prolonged time. This information is an essential prereq- uisite for developing new implantable aDBS devices for chronic PD treatment.

In this single-case study, we tested whether a portable DBS device we developed is suitable to compare the clinical benefit in a freely moving PD patient induced by either aDBS or cDBS. To do so, after a first experimental session for extracting patient settings to personalize the aDBS algorithm, we treated a blinded patient (51 y old, male, 8 y PD history) with cDBS and aDBS in two separate experimental sessions each lasting $120 \mathrm{~min}, 5$ and $6 \mathrm{~d}$, respectively, after DBS electrode implant. To ensure reliable results, the patient underwent repeated clinical assessments every $20 \mathrm{~min}$ (T1T5) by two independent blinded neurologists through Unified Parkinson's Disease Rating Scale (UPDRS) III subsections and Rush Dyskinesia Rating Scale (see Supplemental Data for details).

The aDBS portable device we used was equipped with an ad hoc algorithm that analyzed patient's LFP beta band power (13-17 Hz) and adapted voltage stimulation linearly each second (Fig. 1A).

The patient during aDBS experienced a more stable condition than during cDBS, with better control of symptoms and dyskinesias over time (Fig. 1; video 1). In particular, aDBS and cDBS improved patient's axial symptoms to a similar extent (Fig. 1B), but compared with cDBS, aDBS significantly improved his main symptom, bradykinesia (Fig. 1C). aDBS did not elicit side effects and was well tolerated.

Because we evaluated the patient a few days after surgery when he probably manifested a stunning effect, ${ }^{5}$ the aDBSand cDBS-induced improvements were lower than those reported by others in follow-up DBS studies. ${ }^{6}$ A major clinical achievement was that compared with cDBS, aDBS greatly reduced the patient's dyskinesias during gait and at rest (Fig. 1B; Fig. 1D). Presumably it did so because we designed the adaptive algorithm to avoid dyskinesias related to hyperstimulation: when L-dopa reduced beta-band LFP activity, the voltage linearly diminished, avoiding hyperstimulation.

(C) 2014 The Authors. Movement Disorders published by Wiley Periodicals, Inc. on behalf of International Parkinson and Movement Disorder Society. This is an open access article under the terms of the Creative Commons Attribution License, which permits use, distribution and reproduction in any medium, provided the original work is properly cited.

*Correspondence to: Dr. Manuela Rosa, MS, Università degli Studi di Milano, Centro Clinico per la Neurostimolazione, le Neurotecnologie ed i Disordini del Movimento Fondazione IRCCS, Ca' Granda Ospedale Maggiore Policlinico, Via Francesco Sforza 35, Milano, 20122 Italy, e-mail: manuela.rosa@policlinico.mi.it

Funding agencies: This study was supported by Fondazione IRCCS Ca' Granda Ospedale Maggiore Policlinico and by Università degli Studi di Milano (Italy).

Relevant conflicts of interest/financial disclosures: Filippo Cogiamanian, Sara Marceglia, Paolo M Rampini e Alberto Priori are shareholders of Newronika s.r.l., a spin-off company of Fondazione IRCCS Ca' Granda Ospedale Maggiore Policlinico, Milan and Università degli Studi di Milano.

Full financial disclosures and author roles may be found in the online version of this article.

Received: 6 October 2014; Revised: 20 February 2015; Accepted: 21 March 2015

Published online 21 May 2015 in Wiley Online Library (wileyonlinelibrary.com). DOI: 10.1002/mds.26241 